\title{
Circuit Design and Power Consumption Analysis of Wireless Gas Sensor Nodes: One-Sensor Versus Two-Sensor Approach
}

\author{
Andrey Somov, Member, IEEE, Alexander Baranov, Denis Spirjakin, \\ and Roberto Passerone, Member, IEEE
}

\begin{abstract}
Wireless sensor networks (WSNs) have recently been applied in industrial monitoring applications including hazardous gases detection. As a major power consumer of a node, gas sensors may significantly constrain its lifetime. Hence, the sensing circuit must be carefully designed to optimize performance and retain accuracy. In this paper, we propose for the first time the principle of gas concentration measurement based on a single sensor in a voltage divider circuit, instead of the well-known Wheatstone bridge sensing circuit, which employs two sensors. We discuss the design of a real WSN node for gas sensing and evaluate it with respect to an identical platform that uses the Wheatstone bridge. The proposed approach ensures significant energy savings and helps to avoid zero offset issue. Besides, we employ a more efficient and secure sensor heating profile; it does not damage the sensor and does not make gas sensing dependent on environmental conditions. Experimental results show a $30 \%$ reduction in power with respect to the state-of-the-art.
\end{abstract}

Index Terms-Catalytic sensor, dual power supply, gas detection, remote sensing, wireless sensor network.

\section{INTRODUCTION}

W IRELESS Sensor Networks (WSN) have recently been applied in various monitoring applications [1]-[5]. WSNs provide a number of benefits over traditional wired sensor systems, since no expensive cable production is required, making deployment and maintenance easier. These advantages are especially relevant in industrial premises where huge spaces imply long distances to be covered [6]. Nonetheless, industrial WSNs are subject to additional requirements and challenges [7], such as reliable communication [8] and long-term unattended operation, along with precise and frequent measurements and fast sensor response [9]. At the

Manuscript received January 24, 2014; accepted February 24, 2014. Date of publication February 27, 2014; date of current version April 23, 2014. This work was supported by the Russian Federal Program Development of Electronic Components and Radio Electronics under Grant 14.429.11.0004. The associate editor coordinating the review of this paper and approving it for publication was Dr. M. R. Yuce.

A. Somov is with the CREATE-NET, Trento 38123, Italy (e-mail: asomov@create-net.org).

A. Baranov and D. Spirjakin are with the MATI-Russian State Technological University, Moscow 121552, Russia (e-mail: baranov@mccinet.ru; denis.spirjakin@gmail.com).

R. Passerone is with the Dipartimento di Ingegneria e Scienza dell'informazione, University of Trento, Trento 38123, Italy (e-mail: roberto.passerone@unitn.it).

Color versions of one or more of the figures in this paper are available online at http://ieeexplore.ieee.org.

Digital Object Identifier 10.1109/JSEN.2014.2309001 same time, wired sensor systems do not suffer from the power consumption limitations of WSNs [10], which significantly restrict the kind and the nature of the components that can be integrated on the nodes, and consequently the extent of the functions that they can perform. This problem is of vital importance for WSNs in the context of fire [11] and hazardous gases [12] monitoring. In fact, in contrast to typical applications [3] where the radio module is the main power sink [13], these applications require on board power hungry sensors (up to $600 \mathrm{~mW}$ ) [14] to meet safety requirements [15] and to avoid human victims and huge pecuniary loss. Energy scavenging methods have been investigated for this kind of systems [16]. However, only direct solar radiation can provide enough power to support gas sensors. Light, acoustic or electromagnetic waves scavenged in indoor industrial premises can provide current in the $\mu \mathrm{A}$ range only [17], which is not sufficient to ensure proper system lifetime.

The hazardous gas concentration is typically measured using film (colorimetric), optical, or catalytic/semiconductor sensors. The first approach is based on the use of a light sensor in conjunction with a colorimetric chemical sensing film [18], [19]. The light intensity reaching the light sensor is modulated by changes in the colour of the sensing film, which indicates the presence of chemical plumes. Wireless sensor nodes of this type are characterized by low power consumption, but long sensor response time (around $300 \mathrm{~s}$ ) that does not meet safety regulations. The second approach is implemented using laser spectroscopic trace-gas sensor platforms [20]. These platforms use laser spectroscopy to detect and quantify numerous gas species at part-per-million to part-per-billion (ppm-ppb) concentrations. Being highly sensitive and fast in terms of sensor response, these systems satisfy safety regulations perfectly. However, circuits of this type may consume more than $500 \mathrm{~mA}$, making them unsuitable for WSN applications. Sensor nodes based on catalytic/semiconductor sensors [14], [21] are a trade-off between film and optical approaches: on one hand, they consume less power than optical nodes, while on the other hand they have much better response time and selectivity in contrast to the film approach.

Circuits for gases detection with catalytic/semiconductor sensors are commonly based on the Wheatstone bridge, which includes two resistors and two sensors, one active and one for reference. Most of the power goes into the sensor heating process, required to perform the measurement. 
In this work, we present a study on power-aware gas sensing using the WSN paradigm. The contribution of this work in terms of power consumption reduction is twofold:

- Sensing circuit: We show how to decrease power consumption by replacing the traditional Wheatstone bridge sensing circuit with a simple voltage divider. In addition, we replace the typically used linear regulator power supply by a more efficient DC/DC converter and improve the quality of the measured signal by integrating a filter and an amplifier circuit.

- Sensor heating profile: We apply a four-stage heating profile (in contrast to the widely used continuous or simple two-stage pulse (heat or not) heating in Wheatstone bridge sensing circuits) to drive the sensor through the simple voltage divider. This helps to further reduce the power consumption of the WSN node and does not damage the sensor during the frequent heating-cooling processes [14].

To show the advantages of our approach in terms of power consumption reduction, we develop and compare two different WSN platforms for industrial applications and measurement techniques using a catalytic sensor, one based on our proposed single sensor architecture, and the other on the traditional twosensor Wheatstone bridge sensing circuits. Our results show that the designed platform consumes $30 \%$ less power than the best platform that has been reported in the literature, for an increased lifetime of the system.

This paper is structured as follows. After reviewing the operating principles of the sensing circuits, Section II discusses the design of the WSN platform for gas and describes its operation. Section III then presents experimental results in terms of measured power consumption and sensor response. This evaluation is done for both sensing platforms. State-of-the art analysis is conducted in Section IV. Finally, we provide concluding remarks.

\section{Sensor Node Design}

In this section, we first review two circuits for measuring the output of catalytic gas sensors, and then discuss how they can be integrated into a complete WSN platform. Finally, we provide an analytical assessment of the sensing performance of our proposed solution.

\section{A. Sensing Circuits}

Catalytic sensors are most used in the Lower Explosive Limit (LEL) gas concentration range due to their high sensitivity, selectivity, linear and fast response (300 ms in our case) and low cost. The power consumption of the catalytic sensor used in this work is $120 \mathrm{~mW}$ in continuous heating mode. In contrast, semiconductor sensors are highly sensitive in the Parts per Million (PPM) range, a range not relevant for combustible gases detection. The reader can find more details on sensor technologies comparison in [23] and [24].

The working principle of a catalytic sensor consists in flameless burning (oxidation) of combustible gas on the surface of a catalytically active substance. To ensure gas

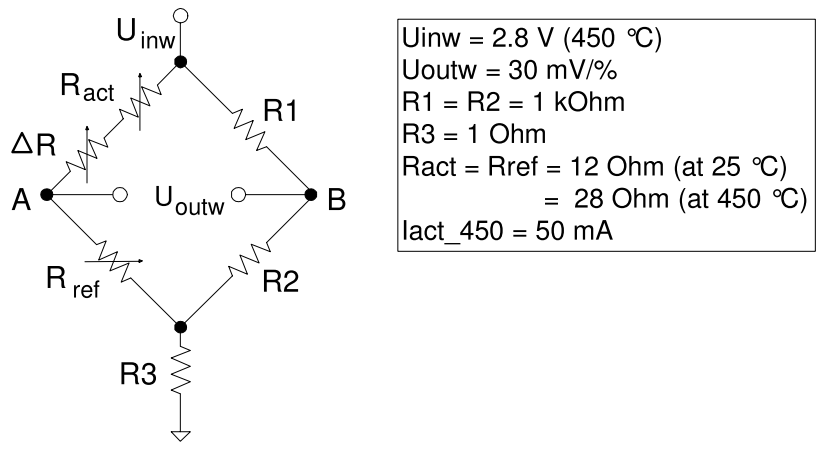

(a)

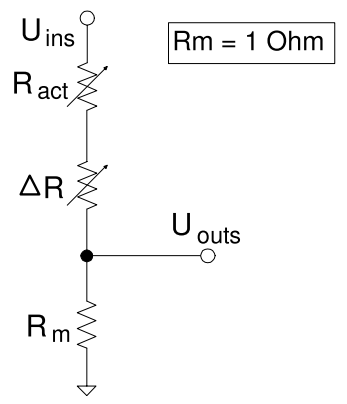

(b)

Fig. 1. Detailed sensing circuit schematic. (a) Wheatstone. (b) Differential.

burning, the catalyst must be heated up to a temperature that, for methane monitoring, is in the range of 400 to $450{ }^{\circ} \mathrm{C}$. The heat generated by burning is proportional to the combustible gas concentration, and changes the temperature of the sensor. In turn, the sensor resistance depends on the temperature as:

$$
\Delta R=R_{0} \cdot(1+\alpha \cdot \Delta T)
$$

where $R_{0}$ is the resistance at $T=25^{\circ} \mathrm{C}, \alpha$ is a temperature resistance coefficient, and $\Delta T$ is the temperature change. Thus, we can estimate the gas concentration by tracking the resistance change of the sensor.

Typically, the change in resistance $\Delta R$ is measured using a Wheatstone bridge [see Fig. 1(a)], which includes two resistors, $R 1$ and $R 2$, and two sensors, one active, $R_{a c t}$, and one for reference, $R_{\text {ref }}$. $R 3$ is a current shunt (optional) resistor to measure the heating current. The active sensor, which uses the catalytic material, is used to perform the measurement. The reference sensor, which is identical to the active sensor but not covered by the catalyst and therefore insensitive to the gas concentration, is instead used to compensate for environmental factors such as temperature and humidity. This circuit is very reliable. However, a number of disadvantages prevent its application in WSNs: (i) it has inherent high power consumption, since there are two sensors [14], and (ii) it requires frequent 'zero offset' calibrations, since the parameters of the two sensors vary by unequal amounts with respect to each other over time.

In this work we propose and implement a differential technique with only one catalytic sensor [22] needed for 


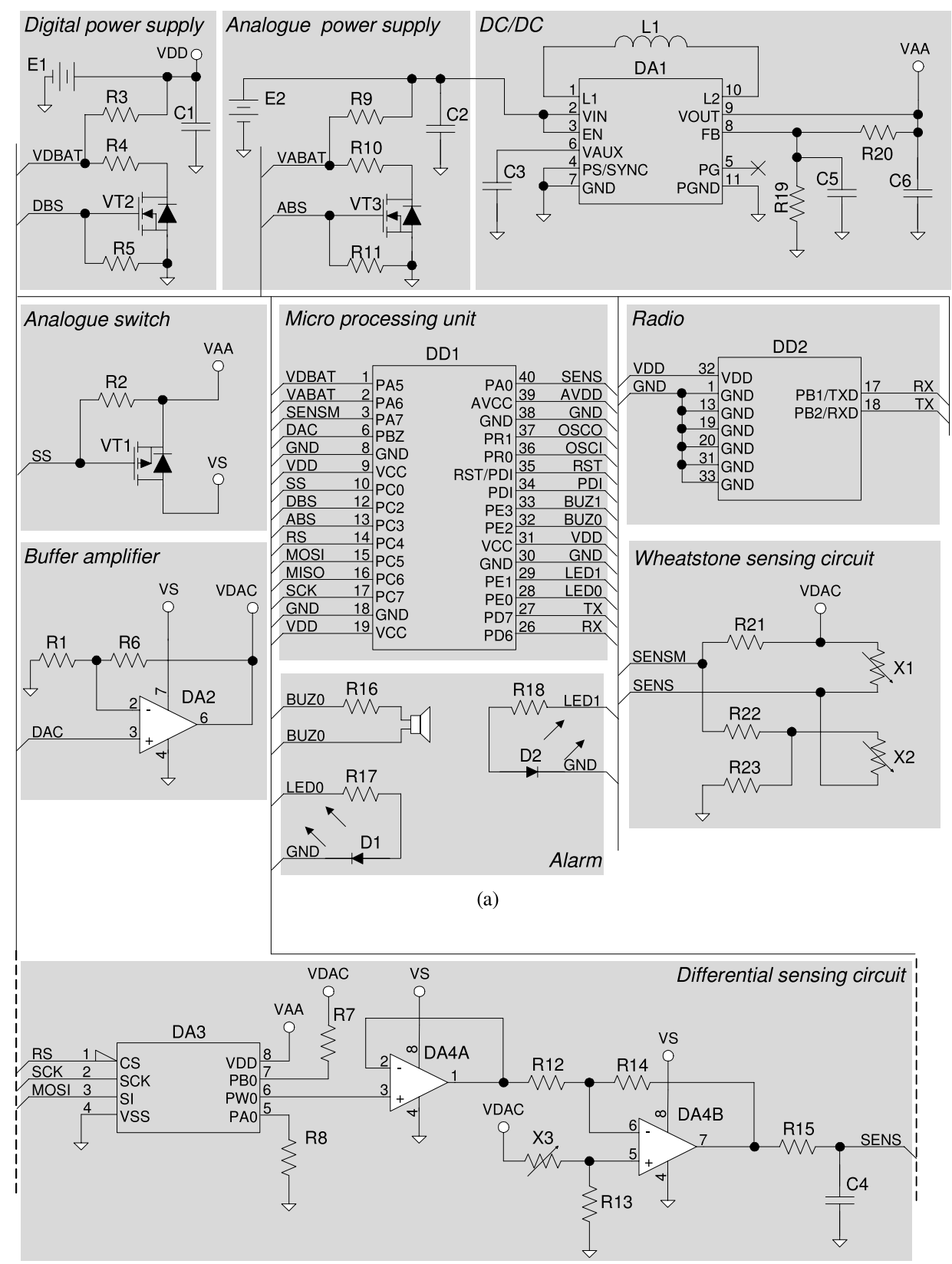

(b)

Fig. 2. (a) Sensor node platform with Wheatstone sensing circuit and (b) differential sensing circuit which can replace the Wheatstone block in the circuit schematic (a).

gas measurement. The catalytic sensor, $R_{a c t}$, forms one arm of a voltage divider circuit [Fig. 1(b)]. Resistance $R_{m}$ is a known measured precise resistor needed to calculate the divider parameters. The sensor is initially heated up till the very beginning of burning of combustible gas on the catalyst surface $\left(200{ }^{\circ} \mathrm{C}\right)$. At this temperature the oxidation of methane does not start, but moisture evaporates from the sensing layer. A measurement is taken at this temperature as the reference point. Next, the sensor is heated up to the working temperature at $400-450{ }^{\circ} \mathrm{C}$ when the gas starts to burn, and a second measurement is taken. Since environmental parameters equally affect both measurements, their influence can automatically be excluded. Besides, since the measurements are conducted with only one sensor at different temperatures, zero offset calibration is not required. We assess both sensing circuits in more details in Section III.

\section{B. Platform Design}

The circuit schematic of a sensor node based on the Wheatstone bridge is shown in Fig. 2(a).

1) Micro Processing Unit: The core element of the node is the DD1 ATxmega32A4 microcontroller (MCU), chosen due to its low power consumption $(\sim 1 \mu \mathrm{A}$ in power-save mode), sufficient performance and high-precision 12-bit ADC and DAC. The MCU performs, among other 
functions, circuit management, controls the sensors heating, processes the measured ambient conditions, and interfaces with the radio module.

2) Radio: To provide communication across the WSN, we have used a DD2 ETRX357 ZigBee transceiver. It complies with the IEEE 802.15.4 standard, operates in the $2.4 \mathrm{GHz}$ ISM band and has a low sleep current $(\sim 0.7 \mathrm{uA})$. Both devices support a wide supply voltage range from $2.1 \mathrm{~V}$ to $3.6 \mathrm{~V}$.

3) Sensor: In this work we use a planar catalytic sensor (prototype) [26] manufactured by NTC-IGD, Russia, for hazardous gases detection. The sensor is fabricated by planar technology on nano-porous gamma anodic alumina membrane with thickness $30 \mu \mathrm{m}$. Micro-heaters are fabricated by magnetron sputtering of a platinum target and covered by thin film layer of $\mathrm{Al}_{2} \mathrm{O}_{3}$ to prevent its degradation. The heated area is about $200 \times 200 \mu \mathrm{m}^{2}$. To obtain the catalytic activity the porous gamma alumina membrane is impregnated with catalytic metals (Pd and $\mathrm{Pt}$ ). This sensor is calibrated by the manufacturer and is provided with the specification indicating the calibration values. These values can be programmed into the memory of the sensor node when the sensor degrades with time (1-1.5 year). The sensor can be calibrated to detect various hazardous gases, e.g., hydrocarbon based gases and $\mathrm{H}_{2}$. The signal required to heat the sensor(s) must be generated according to a specific profile, described below, to guarantee the proper operation of the sensing circuit. The profile is generated by the software running on the MCU, using the integrated Digital to Analog Converter (DAC). This signal, at a reference value of $1 \mathrm{~V}$, is buffered by an AD8513R amplifier (DA2), which provides sufficient current (not available at the MCU outputs) and voltage (the gain is set to 3). The output voltage from the buffer amplifier is supplied to the sensors $X 1-X 2$. Conversely, the measured sensor response in volts is supplied directly to the ADC of the MCU, and is acquired by the node under software control. We note that proposed platform is compatible with any catalytic and semiconductor sensors which have similar specification to our sensor [26].

4) Power Supply: The platform components are divided into a digital (MCU, radio, alarm) and an analog section (sensing, buffer), operated by separate power supplies. The digital power supply $E 1$ consists of a AA-sized $\mathrm{Li}-\mathrm{SOCl}_{2}$ cell with a nominal capacity of 2.6 Ah. In contrast to previous work [22], which used three C-sized, $1.5 \mathrm{~V}$ alkaline cells wired in seriesand a linear regulator to provide the sensing circuit with a stable heating voltage of $3 \mathrm{~V}$, in this work we use two AA-sized, $3.3 \mathrm{~V}, 3000 \mathrm{mAh}$ lithium cells $E 2$ wired in parallel and use a DC/DC TPS63030 converter (DA1). This solution improves the operation of the sensor node due to its high conversion efficiency (98\%) and high energy density. The analog part of the circuit can be switched off using an analog switch based on MOSFET VTI. Such design prevents digital noise from entering the analog circuit. In addition, it provides high reliability and long lifetime because the average sensor power consumption exceeds the digital circuit consumption. Thereby, in case of discharge of the first battery, the node does not disconnect from the WSN and can transmit alert messages.

A WSN node that uses the differential technique [Fig. 2(b)] can be derived from the previous one by replacing the

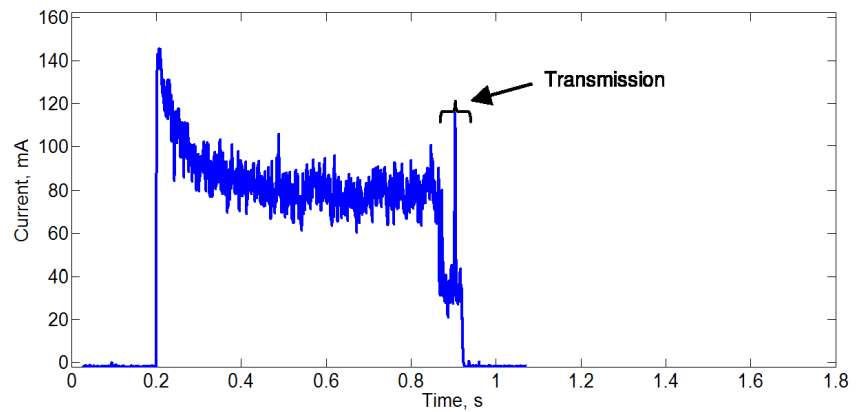

Fig. 3. Current consumption diagram for the Wheatstone circuit (sensing: 0.2-0.85 s, data transmission: 0.85-0.95 s).

Wheatstone sensing circuit [Fig. 2(a)], as discussed in the previous section. The circuit with one sensor $X 3$ shown in Fig. 2(b) is more energy efficient, but has worse response. For this reason, the measured voltage is fed into a signal conditioning block, which improves the quality of the measurement. We discuss these aspects next.

\section{Sensor Node Operation}

One requirement for industrial sensor networks is reliable communication [7]. To guarantee energy saving, the node performs data transmission to the gateway only in case when the detected gas concentration exceeds a dangerous threshold, i.e. it avoids unneeded transmissions (we discuss this point later in this section). In all other cases, the sensor node sends unicast messages every 5 minutes to inform the gateway about the node status and correct operation. After successful transmission of the packets, the node immediately goes in sleep mode where it remains for $30 \mathrm{~s}$. To avoid latency and delays of the packet transmission from the nodes to the gateway over a multi-hop network, we assume that the network topology is of star configuration. This is done due to the critical nature of the application to improve the communication reliability.

If the node detects a non-zero gas concentration below $0.15 \%$ vol., it notifies the user "locally" using LED D1 and $D 2$ and/or a sound alert (buzzer). If the gas concentration is between $0.15 \%-0.5 \%$ vol., the node sends an alarm message to the network coordinator [14]. When the detected concentration is between $0.5 \%-1 \%$ vol., the node activates a wireless actuator to close the gas valve, in addition to sending the alert message. The lower limit for methane explosion is 5\% vol.

\section{EXPERIMENTAL ReSUlTS AND Discussion}

In this section, we compare both circuits by conducting a number of experiments and discuss the obtained results.

\section{A. Power Consumption Analysis}

The goal of this section is to evaluate and to compare the two sensing circuits designed in Section II. For this purpose we evaluate the heating profile lengths and power consumption.

Figs. 3 and 4 show the current consumption of the node with the Wheatstone bridge and with the differential technique, 


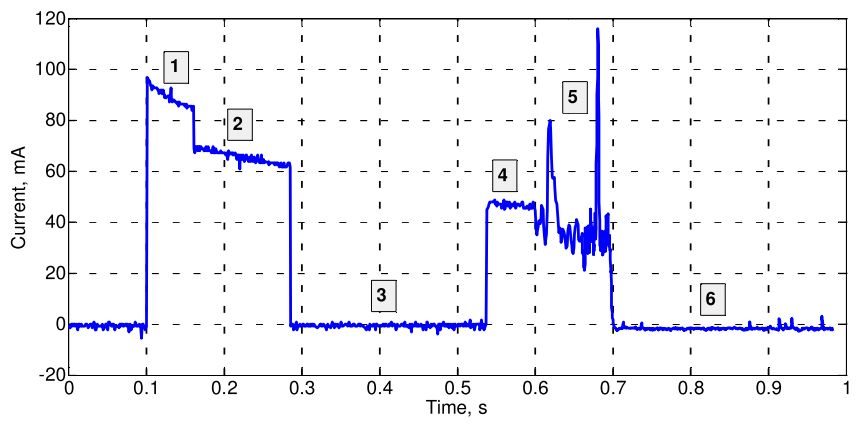

Fig. 4. Current consumption diagram for the voltage divider where " $1 "$ - is sensor heating pulse, " $2 "$ - is a temperature support pulse, " $3 "$ nothing happens during this time period, " 4 " - is the final heating pulse to guarantee moisture evaporation from sensor's surface, "5" - data transmission pulse (0.604-0.704 s), "6" - sensor sleep mode: it is awaiting for next measurement cycle.

respectively. In the Wheatstone bridge case, a heating voltage of $2.8 \mathrm{~V}$ is used to heat the sensor(s) up to the operational temperature $\left(450{ }^{\circ} \mathrm{C}\right)$. Conversely, the differential circuit requires a complex 4-pulse heating profile with different heating voltage amplitudes and durations.

The heating time for the sensors in the Wheatstone circuit is $0.65 \mathrm{~s}$. The average (constant) current flowing through $R 3$ [see Fig. 1(a)] during heating is $81 \mathrm{~mA}$, resulting in $226.8 \mathrm{~mW}$ of sensor average power consumption. As seen in Fig. 3, the amplitude of the current consumption decreases in time due to increase in the sensor resistivity, as discussed in Section II.

The heating time for the sensor in the differential approach is $0.504 \mathrm{~s}$. More precisely, the heating pulse $\mathcal{N} \underline{\mathrm{o}} 1$ is $55 \mathrm{~ms}$ at $3.3 \mathrm{~V}$ (current consumption decreases in time as in the case with the Wheatstone circuit), "support" pulse $\mathcal{N}_{\mathrm{O} 2}$ is $145 \mathrm{~ms}$ at $2.4 \mathrm{~V}$, pulse $\mathcal{N} \mathrm{o} 3$ is $244 \mathrm{~ms}$ at $0 \mathrm{~V}$, and the final evaporation pulse $\mathcal{N} \mathrm{o} 4$ is $60 \mathrm{~ms}$ at $1.6 \mathrm{~V}$. This complicated heating profile does not negatively affect the sensor performance or reliability, but results in significant power savings. The average heating current is $30.6 \mathrm{~mA}$ that results in $85.68 \mathrm{~mW}$ of sensor average power consumption. We compare the results of our approach with respect to the state-of-the-art works in Section IV.

\section{B. Sensor Response Assessment}

Consider the Wheatstone circuit shown in Fig. 1(a), where $U_{\text {inw }}$ is the heating voltage, $U_{\text {outw }}$ the sensors response, and $I_{a c t} 450$ is the current flowing through $R_{a c t}$ at $450{ }^{\circ} \mathrm{C}$. Due to the oxidation reaction, $R_{a c t}$ increases by $\Delta R$. For a $1 \%$ methane concentration, the measured output voltage (response) $U_{\text {outw }}$ is $30 \mathrm{mV}$ and $I_{\text {act }} 450$ is $50 \mathrm{~mA}$ (for the analog circuit). Therefore, we can approximately establish that $\Delta R=U_{\text {outw }} / I_{\text {act } \_450}=0.6 \mathrm{Ohm}$.

To achieve the same heating current for the single sensor circuit shown in Fig. 1(b), the heating voltage $U_{\text {ins }}$ must be larger than $U_{\text {inw }} / 2$ (we are using only one measuring arm) because $R_{m}$ reduces the flowing current. $R_{m}$ should be as low as possible since it also dissipates power. Since the final temperature of the sensor is still $450{ }^{\circ} \mathrm{C}$, the resistance change in the presence of a $1 \%$ concentration is still $\Delta R=0.6 \mathrm{Ohm}$. The output voltage at $200{ }^{\circ} \mathrm{C}$ (when the methane oxidation has not started yet) for the single sensor circuit $U_{\text {outs_2 } 200}$ is

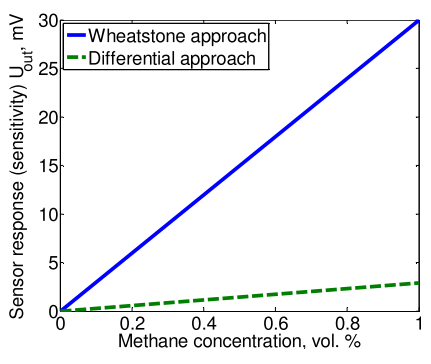

(a)

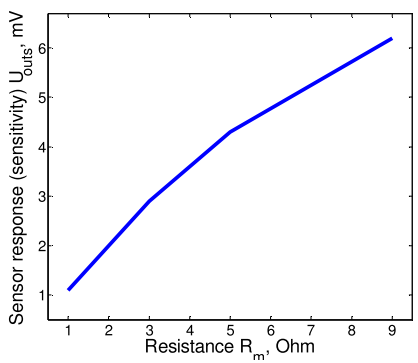

(b)
Fig. 5. Sensor(s) response: (a) comparison of Wheatstone bridge and differential approaches and (b) w.r.t. precise $R_{m}$ resistor in differential approach for $1 \%$ methane concentration.

given by (1):

$$
U_{\text {outs } \_200}=\frac{U_{\text {ins }} \cdot R_{m}}{\left(R_{\text {act }}+R_{m}\right)}
$$

The output voltage at $450{ }^{\circ} \mathrm{C} U_{\text {outs__ } 450}$ is given by (3):

$$
U_{\text {outs } \_450}=\frac{U_{\text {ins }} \cdot R_{m}}{\left(R_{\text {act }}+\Delta R+R_{m}\right)}
$$

The resulted voltage response $U_{\text {outs }}$ is the voltage difference $U_{\text {outs_ } 200}-U_{\text {outs_ }} 450$.

Fig. 5(a) shows the sensor response of the two approaches and Fig. 5(b) shows how the sensitivity of the differential approach depends on the precise resistor $R_{m}$ up to $9 \mathrm{Ohm}$ at $U_{\text {ins }}=1.6 \mathrm{~V}$. We do not consider higher values of $R_{m}$ since they result in higher power consumption. Thus, while the single sensor circuit consumes less power than the Wheatstone circuit, it has a worse (10-30 times) voltage response [see Fig. 5(a)]. In order to digitize this signal with the 12-bit ADC integrated in the MCU we must amplify its value. The sensor response is supplied to the positive input of operational amplifier DA4B OPA2340 that amplifies it by a factor of 100 [see Fig. 2(b)]. A bias voltage is supplied to the negative input of the operational amplifier to exclude the constant component of the measured signal. The bias voltage is generated by resistive divider $R 7, R 8$ and digital potentiometer $D A 3$ MCP41010. The bias voltage is then amplified by the operational amplifier $D A 4 A$. The resulting signal (bias voltage from $D A 4 A$ and amplified voltage from $D A 4 B$ ) is supplied to the ADC of the MCU. This solution helps to achieve the same sensitivity as in Wheatstone circuit.

\section{STATE-OF-THE-ART}

When designing an energy efficient embedded system like a wireless sensor node with a power-hungry sensor, designers typically focus their attention on energy efficient sensing technologies, relevant sensing platforms, and intelligent approaches, which can help further decrease the device power consumption. Since overviews of sensor technologies are widely available in the literature [23] and [24], in this section we discuss power consumption of gas WSN platforms relevant to our work and overview the intelligent approaches proposed recently. 
TABLE I

Power Consumption of State-of-The-Art Gas Sensing Circuits: Comparative STudy

\begin{tabular}{l|l|l|l|l|l|l|l}
\hline \hline & Ho [20] & Wobscholl [27] & FlyPort [28] & Somov 2011 [14] & Yokosawa [21] & Somov 2012 [22] & This work \\
\hline Application & $\begin{array}{l}\text { Hazardous } \\
\text { gases detection }\end{array}$ & $\begin{array}{l}\text { Toxic gases } \\
\text { monitoring }\end{array}$ & $\begin{array}{l}\text { LPG, } \\
\text { natural/town gas }\end{array}$ & $\begin{array}{l}\text { Combustible gases } \\
\text { monitoring }\end{array}$ & $\begin{array}{l}\text { Hydrogen } \\
\text { monitoring }\end{array}$ & $\begin{array}{l}\text { Hazardous gases } \\
\text { monitoring }\end{array}$ & $\begin{array}{l}\text { Hazardous gases } \\
\text { monitoring }\end{array}$ \\
\hline $\begin{array}{l}\text { Sensing power } \\
\text { consumption }\end{array}$ & $1550 \mathrm{~mW}$ & $1000 \mathrm{~mW}$ & $800 \mathrm{~mW}$ & $264 \mathrm{~mW}$ & $200 \mathrm{~mW}$ & $124.30 \mathrm{~mW}$ & $85.68 \mathrm{~mW}$ \\
\hline Response time & N/A & up to $110 \mathrm{~s}$ & up to $10 \mathrm{~s}$ & $1.50 \mathrm{~s}$ & up to $1.20 \mathrm{~s}$ & $\begin{array}{l}0.70 \mathrm{~s}(2 \mathrm{sensors}) \\
0.56 \mathrm{~s}(1 \mathrm{sensor})\end{array}$ & $\begin{array}{l}0.65 \mathrm{~s}(2 \mathrm{sensors}) \\
0.50 \mathrm{~s}(1 \mathrm{sensor})\end{array}$ \\
\hline Sensor & $\begin{array}{l}\text { Laser } \\
\text { spectroscopic }\end{array}$ & $\begin{array}{l}\text { Catalytic/ } \\
\text { semiconductor }\end{array}$ & Semiconductor & Semiconductor & FET & Catalytic & Catalytic \\
\hline Features & $\begin{array}{l}\text { Detects any } \\
\text { gases, accurate } \\
\text { measurements, } \\
\text { high power } \\
\text { consumption }\end{array}$ & $\begin{array}{l}\text { Automatic } \\
\text { calibration }\end{array}$ & $\begin{array}{l}\text { Inaccurate } \\
\text { measurements, } \\
\text { frequent } \\
\text { calibration } \\
\text { required }\end{array}$ & $\begin{array}{l}\text { Wheatstone circ., } \\
\text { accurate } \\
\text { measurements }\end{array}$ & $\begin{array}{l}\text { Long sensor } \\
\text { response }\end{array}$ & $\begin{array}{l}\text { cifferential } \\
\text { measurements, } \\
\text { no calibration } \\
\text { required }\end{array}$ & $\begin{array}{l}\text { Differential } \\
\text { circuit, accurate } \\
\text { measurements, } \\
\text { no calibration } \\
\text { required }\end{array}$ \\
\hline \hline
\end{tabular}

\section{A. Power Consumption of Sensing Circuit}

The results of this work in terms of power consumption of the sensing circuit significantly improve the state-of-the art on hazardous gas detection. In Table I we highlight main features of relevant works and compare them numerically in terms of power consumption of sensing circuit and sensor response time instead of comparing the gas WSN nodes' lifetime as it is done in some papers [23]. Lifetime is not a correct metric for this evaluation due to its strong dependence on power supply type/capacity/etc (AA rechargeable batteries can significantly vary in terms of capacity from $1500 \mathrm{mAh}$ to $3300 \mathrm{mAh}$ ) and operation of radio modules which also vary in terms of power consumption, functionality, and algorithms of operation.

The node proposed by Ho based on laser spectroscopic [20] can accurately and quickly detect all types of hazardous gases, but consumes up to $1550 \mathrm{~mW}$. The Wobscholl [23] and Flyport [28] nodes are based on catalytic and/or semiconductor sensors, consume less power - up to 1000 and $800 \mathrm{~mW}$ respectively, but are designed for specific gases. The Wobscholl node guarantees automatic calibration, whereas Flyport requires frequent calibration and may result in inaccurate measurements, since it ignores environmental effects. The WSN node proposed by Somov et al. [14] largely improves on these, consuming only $264 \mathrm{~mW}$, due to the pulse heating profile instead of the constant heating current. However, the pulse heating profile in this work is a straightforward approach which consists in frequent turning on/off (depending on duty cycle) of heating pulses. This approach helps to reduce the power consumption, but has a number of drawbacks: (i) moisture does not fully evaporate from the surface, which may lead to inaccurate measurements, (ii) real sensors can be quickly damaged suffering from frequent and drastic heating pulses variations, i.e., when heating is on or off. The heating profile presented in this work is designed in a way to fully evaporate the moisture from the sensor's surface and to guarantee sensor long time operation. Yokosawa [21] demonstrates a similar sensor node, which consumes $200 \mathrm{~mW}$, but has long sensor response. To the best of our knowledge, the gas sensor node based on catalytic sensor presented in [22] has the lowest power consumption, namely $124.3 \mathrm{~mW}$. This was achieved by using (i) a sensor implemented by advanced power consumption friendly planar technology and (ii) pulse heating profile. The main emphasis of the work in [22] is on power consumption reduction without the analysis of sensor response. Our work performs this analysis and goes below $100 \mathrm{~mW}$ in terms of power consumption, namely $85.68 \mathrm{~mW}$, by applying the described differential circuit, improved hardware (power management and sensing circuits), and reduced sensor heating pulses. The main advantage of our circuit is that it meets safety requirements [7], [15], ensures the node's long lifetime while guaranteeing good performance.

\section{B. Intelligent Approaches for Power Saving}

Alternative ways for energy efficient gas sensing with WSNs are grounded on intelligent approaches.

The core idea of context-adaptive approaches is to equip a commercial WSN gas sensing platform with a Pyroelectric Infrared (PIR) sensor. The PIR sensor collects the information on people presence to adapt gas measuring frequency. A WSN implementing this approach was deployed in a four-story building and demonstrated good potential towards energy efficiency [23]. However, while demonstrating good performance, the experiments (in contrast to our work) are carried out with no reference to any standards [15] making them not useful in practice.

A similar context aware approach is proposed in [29]. The authors employ the Internet of Things paradigm and cognitive technologies for fire detection. The bottom line of this approach is to use two heterogeneous WSNs interchangeably depending on context. Since the fire detection task can be reduced to pyrolysis sensing $\left(\mathrm{H}_{2}+\mathrm{CO}\right)$ [31] or temperature and humidity control - two heterogeneous WSNs (in terms of onboard sensors and their power consumptions) are deployed in a house. To implement this idea, the authors propose a framework where virtual entities of real objects (nodes) are created and enriched with context information to facilitate the inference procedures. Depending on the situation (people presence, time of the day) and utility cost of the nodes, one of the WSNs is in active mode to ensure reliable fire detection while ensuring safe monitoring. The authors do not provide the 
performance analysis of WSNs focusing more on framework performance.

The WSN node with on-board intelligence developed in [30] makes an inference procedure using collected data. This approach is based on a simple sensor fusion component developed to locally detect chemical species presence, simultaneously estimating their concentration. Making this analysis, the node can decide whether the data contains useful information on dangerous situation, e.g., gas leak, and apply sensor censoring. Our sensor nodes do similar analysis on-board to avoid unneeded transmission by comparing the measured values against a threshold.

The intelligent approaches discussed in this section demonstrate great potential towards enabling power savings, but most of them do not follow safety standards [23], [30] and have not been deployed in real settings that makes them subject to additional evaluation in real conditions.

At the same time, intelligence can be integrated into hardware power management techniques, making them more powerful. Moreover, the Internet of Things paradigm together with cognitive technologies used in [29] can potentially help WSNs to perform self-management, context-aware sensing, and local decision making.

\section{CONClusion}

In this work we have addressed the problem of power consumption reduction in WSNs used in gas sensing applications. In particular, we have demonstrated how to significantly decrease the power consumption of a gas sensor node up to $85.68 \mathrm{~mW}$ in continuous measurement mode. We have proposed and implemented a differential sensor heating profile requiring only one sensor in a voltage divider circuit. The heating profile is a four-stage pulse, which consumes less energy than the continuous and simple two-stage pulse (heat or not) heating approaches. At the same time, the proposed profile prevents the sensor from being damaged as it could happen with a simple pulse heating profile. Additional gain in energy was achieved through careful hardware design, employing efficient DC/DC conversion and signal conditioning. A dual power supply design avoids the sensor node full de-energizing, giving the node the ability to notify the loss of sensing power to the network coordinator.

Besides this, the proposed approach opens up wide vista for secure and energy efficient gas sensing applications using WSN not only because of the gain in power. Apart from the significant energy saving of the sensing circuit, the proposed solution makes gas sensing insensitive to environmental conditions (like in Wheatstone circuit) has the same response as in Wheatstone circuit and, more importantly, does not face the problem of 'zero offset' due to the application of only one sensor. These advantages make our approach especially relevant for industrial applications where lifetime, easy maintenance, and sensing that meets safety requirements are of vital importance.

Our future work includes further investigation of differential circuit and the deployment of sensor nodes in real settings.

\section{REFERENCES}

[1] T. Torfs et al., "Low power wireless sensor network for building monitoring," IEEE Sensors J., vol. 13, no. 3, pp. 909-915, Mar. 2013.

[2] P. Bellavista, G. Cardone, A. Corradi, and L. Foschini, "Convergence of MANET and WSN in IoT urban scenarios," IEEE Sensors J., vol. 13, no. 10 , pp. 3558-3567, Oct. 2013.

[3] X.-H. Cao, J.-M. Chen, Y. Xiao, and Y.-X. Sun, "Building-environment control with wireless sensor and actuator networks: Centralized versus distributed," IEEE Trans. Ind. Electron., vol. 57, no. 11, pp. 3596-3605, Nov. 2010.

[4] H. Yang, Y. Qin, G. Feng, and H. Ci, "Online monitoring of geological $\mathrm{CO}_{2}$ storage and leakage based on wireless sensor networks," IEEE Sensors J., vol. 13, no. 2, pp. 556-562, Feb. 2013.

[5] L. Mottola, G. P. Picco, M. Ceriotti, S. Guna, and A. L. Murphy, "Not all wireless sensor networks are created equal: A comparative study on tunnels," ACM Trans. Sens. Netw., vol. 7, no. 2, pp. 1-33, Sep. 2010.

[6] Assessment Study on Sensors and Automation in the Industries of the Future, U.S. Dept. Energy, Washington, DC, USA, 2004.

[7] V. C. Gungor and G. P. Hancke, "Industrial wireless sensor networks: Challenges, design principles, and technical approaches," IEEE Trans. Ind. Electron., vol. 56, no. 10, pp. 4258-4265, Oct. 2009.

[8] G. Franchino and G. Buttazzo, "WBuST: A real-time energy-aware MAC layer protocol for wireless embedded systems," in Proc. ETFA, 2012, pp. 1-8.

[9] K. Al Agha et al., "Which wireless technology for industrial wireless sensor network? The development of OCARI technology," IEEE Trans. Ind. Electron., vol. 56, no. 10, pp. 4266-4278, Oct. 2009.

[10] J. Haase, J. M. Molina, and D. Dietrich, "Power-aware system design of wireless sensor networks: Power estimation and power profiling strategies," IEEE Trans. Ind. Inf., vol. 7, no. 4, pp. 601-613, Nov. 2011.

[11] P. Cheong, K. Chang, Y. Lai, S. Ho, I. Sou, and K. Tam, "A zigbeebased wireless sensor network node for ultraviolet detection of flame," IEEE Trans. Ind. Electron., vol. 58, no. 11, pp. 5271-5277, Nov. 2011.

[12] A. Somov, A. Baranov, D. Spirjakin, A. Spirjakin, V. Sleptsov, and R. Passerone, "Deployment and evaluation of a wireless sensor network for methane leak detection," Sens. Actuators A, Phys., vol. 202, no. 11 pp. 217-225, Nov. 2013.

[13] R. C. Luo and O. Chen, "Mobile sensor node deployment and asynchronous power management for wireless sensor networks," IEEE Trans. Ind. Electron., vol. 59, no. 5, pp. 2377-2385, May 2012.

[14] A. Somov, A. Baranov, A. Savkin, D. Spirjakin, A. Spirjakin, and R. Passerone, "Development of wireless sensor network for combustible gas monitoring," Sens. Actuators A, Phys., vol. 171, no. 2, pp. 398-405, Jul. 2011.

[15] Electrical Apparatus for the Detection of Combustible Gases in Domestic Premises, EN Standard 50194:2000, 2000.

[16] S. A. Oulette and M. D. Todd, "Cement seawater battery energy harvester for marine infrastructure monitoring," IEEE Sensors J., vol. 14, no. 3, pp. 865-872, Mar. 2014.

[17] C. O. Mathuna, T. O’Donnell, R. V. Martinez-Catala, J. Rohan, and B. O'Flynn, "Energy scavenging for long-term deployable wireless sensor networks," Talanta J., vol. 75, no. 3, pp. 613-623, May 2008.

[18] J. Hayes, S. Beirne, K.-T. Lau, and D. Diamond, "Evaluation of a low cost wireless chemical sensor network for environmental monitoring," in Proc. Sensors, 2008, pp. 530-533.

[19] Y. W. Kim, S. J. Lee, G. H. Kim, and G. J. Jeon, "Wirelss electronic nose network for realtime gas monitoring system," in Proc. ROSE, 2009, pp. 169-172.

[20] S. Ho, F. Koushanfar, A. Kosterev, and F. Tittel, "LaserSPECks: Laser spectroscopic trace-gas sensor networks-sensor integration and application," in Proc. IPSN, 2007, pp. 226-235.

[21] K. Yokosawa, K. Saitoh, S. Nakano, Y. Goto, and K. Tsukada, "FET hydrogen-gas sensor with direct heating of catalytic metal," Sens. Actuators B, Chem., vol. 130, no. 1, pp. 94-99, Mar. 2008.

[22] A. Somov et al., Energy-Aware Gas Sensing Using Wireless Sensor Networks (Lecture Notes in Computer Science). New York, NY, USA: Springer-Verlag, 2012, pp. 245-260.

[23] V. Jelicic, M. Magno, D. Brunelli, G. Paci, and L. Benini, "Contextadaptive multimodal wireless sensor network for energy-efficient gas monitoring," IEEE Sensors J., vol. 13, no. 1, pp. 328-338, Jan. 2013.

[24] X. Liu, S. Cheng, H. Liu, S. Hu, D. Zhang, and H. Niug, "A survey on gas sensing technology," Sensors, vol. 12, no. 7, pp. 9635-9665, Jul. 2012.

[25] N. Baccour et al., "Radio link quality estimation in wireless sensor networks: A survey," ACM Trans. Sens. Netw., vol. 8, no. 4, pp. 1-33, Sep. 2012 
[26] E. E. Karpov et al., "Energy efficient planar catalytic sensor for methane measurement," Sens. Actuators A, Phys., vol. 194, no. 1, pp. 176-180, May 2013.

[27] D. Wobscholl, "A wireless gas monitor with IEEE 1451 protocol," in Proc. SAS, 2006, pp. 162-164.

[28] (2013). Flyport Sensing Platform [Online]. Available: http://www.openpicus.com

[29] D. Kelaidonis et al., "Virtualization and cognitive management of real world objects in the internet of things," in Proc. GreenCom, 2012, pp. 187-194.

[30] S. De Vito et al., "Wireless sensor networks for distributed chemical sensing: Addressing power consumption limits with on-board intelligence," IEEE Sensors J., vol. 11, no. 4, pp. 947-955, Apr. 2011.

[31] A. Somov et al., "Combustible gases and early fire detection: An autonomous system for wireless sensor networks," in Proc. e-Energy, 2010, pp. 85-93.

Andrey Somov received the B.S. and Diploma degrees in electronics engineering from Moscow State Aviation Technological University, Russia, in 2004 and 2006, respectively. Before starting the Ph.D., he was an Electronics Engineer of Space Technology for two years. He subsequently undertook research in the area of wireless sensor networks in which he received the Ph.D. degree from the University of Trento, Italy, in 2009. In 2008, he was a Visiting Scholar with the University of California, Berkeley, CA, USA, where he conducted research in energy efficient sensor networks. He holds the position of Researcher with CREATE-NET, Italy. His work is focused on the Internet of things and wireless sensor networks.
Alexander Baranov is a Professor with Moscow State Aviation Technological University, Russia. He received the Ph.D. degree in physics and mathematics from the Moscow Institute of Electronics and Mathematics (Technical University) and the Doctor of Technical Science degree from Moscow State Aviation Technological University, in 1994 and 2003, respectively. He is the Project Leader on several research projects, some with International partners. His current research interests include the development of thin-film nanocomposite catalysts for gas sensors by plasma deposition methods, catalytic and semiconductor sensor characterisation, and wireless sensor networks.

Denis Spirjakin received the B.S. and M.S. degrees in electronics from Moscow State Aviation Technological University, Russia, in 2005 and 2007, respectively, where he is currently pursuing the Ph.D. degree at the Department of High Tech Radio-Electronics. His research interests include digital signal processing, data transmission system, and wired/wireless sensor networks.

Roberto Passerone is an Assistant Professor with the Department of Information Engineering and Computer Science, University of Trento, Italy. He received the M.S. and Ph.D. degrees in electrical engineering and computer sciences from the University of California, Berkeley, in 1997 and 2004, respectively. Before joining the University of Trento, he was a Research Scientist with Cadence Design Systems. His current research interests include formal models for system design and the development of design methodologies for embedded systems, with particular attention to wireless sensor networks. 